\title{
Ophthalmologica
}

Abdelkader, E. 60

Aylward, B. 1, 58

Bastawrous, A. 46

Bopp, S. 18

Chandra, A. 3

Charles, S. 27

Charteris, D.G. 3

Heimann, H. 18, 36

Irigoyen, C. 36

Lai, W. 53

Lo, A.C.Y. 10

Lois, N. 60

Morphis, G. 36
Murrary, A. 60

Parkes, C. 46

Prasad, S. 46

Ramalingam, S. 60

Siddiqui, R.M. 60

Smith, J. 42

Stappler, T. 36

Steel, D.H.W. 27, 42

Wong, D. 1, 10, 53

Wong, R.L.M. 10

Woo, T.T.Y. 10

Yorston, D. 3

Yusof, W. 53

\section{Subject Index Vol. 226, Suppl. 1, 2011}

Anterior chamber intraocular lens 46

Aphakia 46

Aspiration 27

Audit 58

Capsular support, inadequate 46

Case mix 58

Caspase 10

Complications 18

Cut rate 27

Dataset 58

Densiron 60

Duty cycle 27

$\mathrm{F}_{6} \mathrm{H}_{8} 60$

Face-down posturing 3

Fluidics 27

Infusion 27

Intraocular lens implant, secondary 46

- -, sclera-fixated 46

Macular hole 3

Mitochondria 10

Necrosis 10

Neuroprotection 10

Outcome 58

Perfluorohexyloctane 60
Perforating diathermy 42

Plaque radiotherapy 42

Posterior chamber intraocular lens 46

Posturing 3

Primary vitrectomy 18

Programmed cell death 10

Proliferative vitreoretinopathy 60

Retinal break 53

- detachment 18,60

- folds 18

Revalidation 58

Scleral buckling surgery 18

Silicone oil 36,60

- - infusion 53

Subretinal fluid drainage 53

Tamponade 36, 60

Transconjunctival cryotherapy 42

Transscleral resection 42

Transvitreal resection 42

Vasoproliferative tumours 42

Vitrectomy 3, 27

Vitreoretinal surgery 36

Vitreous base shaving 53

(C) 2011 S. Karger AG, Basel 